\title{
A sketch of Arnfried Antonius (1934 - 2010)
}

\author{
Sascha C.C. Steiner ${ }^{1,2}$ \\ 1. Trostgasse 16, 2500 Baden, Austria \\ 2. Institute for Tropical Marine Ecology Inc., P.O. Box 944, Roseau, Dominica; scc.steiner@itme.org
}

Received 28-VI-2011. C Corrected 20-IX-2011. Accepted 20-XII-2011.

Arnfried Antonius (Fig. 1) studied Zoology, Paleontology and Ethnology at the University of Vienna, Austria, gradually focusing his attention on the ecology and graphic microanatomical reconstructions of marine invertebrates (Antonius 1965). He completed his Ph.D. in 1967 with a dissertation on convolutid Platyhelminthes from the Red Sea (Antonius 1967). The Marine Biology Institute in Rovinj (then Yugoslavia, now Croatia) and the Museum of Natural History in Venice, Italy, were some of the places where he conducted his work during that time. Arnfried then explored Apulia (Italy), assisting Michele Sarà from the University at Bari, Italy, in search of an adequate location to establish a littoral research field station. Three sites were identified for a final consideration, but Michele Sarà relocated to Genoa and the project was abandoned. Driven to explore and see the world, Arnfried accepted an invitation by the hydrobotanist Fritz Gessner, to work at a new facility in Cumaná, Venezuela, in 1968, and joined the faculty of the Universidad de Oriente. Despite ample funding for the time, the facilities did not have a histology laboratory or microscopes. Arnfried thus started working with "larger" organisms, namely stony corals of the Golfo de Cariaco, where cold-water upwelling made for an interesting environmental setting (Antonius 1968, 1980), and Colombia (Antonius 1972). During the $4^{\text {th }}$ Latin American Zoology

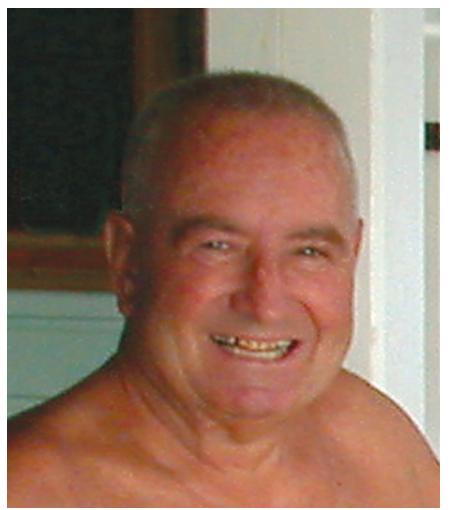

Fig. 1. Arnfried Antonius at Carry Bow Cay in 2001. Detail from a photo by Mike Carpenter.

Congress, held in Caracas in 1968, Arnfried met Jocelyn López Montes de Oca, who was studying chitons at the Universidad Autónoma de Santo Domingo (incidentally the first graduate of marine biology from that institution), and whom he later married. In 1969, when Acanthaster planci outbreaks on coral reefs in the Pacific brought forth panic and funding, Arnfried was invited to join research teams assessing the situation in Ifaluk and Woleei, Micronesia (Antonius 1971).

In 1972 Arnfried visited Klaus Rützler, a fellow graduate from Vienna, at the Smithsonian Institution's National Museum of Natural History in Washington D.C., to examine the 
coral collections. This visit followed previous joint coral reef surveys and developed into a postdoctoral fellowship (Fig. 2). It also marked the beginning of Arnfried's continued involvement in Caribbean marine science, starting with reef surveys off the shore of Belize (then British Honduras) and a historic turn of events involving a briefly disoriented boat crew. Together with Klaus, he was assigned to charter a boat and retrieve an inflatable boat, outboard engine, compressor and other equipment from Glover's Reef. The equipment had previously been deposited there by a group of young, enthusiastic biologists and geologists, convinced that they would receive funding for the establishment of a research facility on one of the Caribbean's few atolls, following extensive surveys and a proposal-development workshop with representatives from approximately 40 institutions (Rützler 2009). Glover's Reef atoll is situated about $20 \mathrm{~km}$ east of the Mesoamerican Barrier Reef, Belize; the group's proposition was to compare it with a Pacific atoll, 10 years down the road. That grant never came through. Having retrieved the equipment, Arnfried and Klaus were returning from Glover's Reef when the boat captain lost his orientation and couldn't find Tobacco Cay Entrance. Instead, he found another cut through the reef, next to which there was a small island. Arnfried, Klaus and the rest of the crew went on land. The island was deserted but there was a sign saying, "Welcome to Carrie Bow Caye". And the rest is history, colloquially put, and has been well documented in Rützler's (2009) summary of the studies that have been carried out at the Smithsonian's Carrie Bow Cay Marine Field Station since its inception in 1972.

In 1973 Arnfried joined the Harbor Branch Foundation in Fort Pierce, Florida. At this time he had already started to address reef degradation of all kinds, supplementing field observations with experimentation and methods of pathology. In the same year he co-founded the Florida Reef Foundation and presented his first article on a "coral killing blue-green alga" at the $10^{\text {th }}$ scientific meeting of the Association of Island Marine Laboratories of the Caribbean

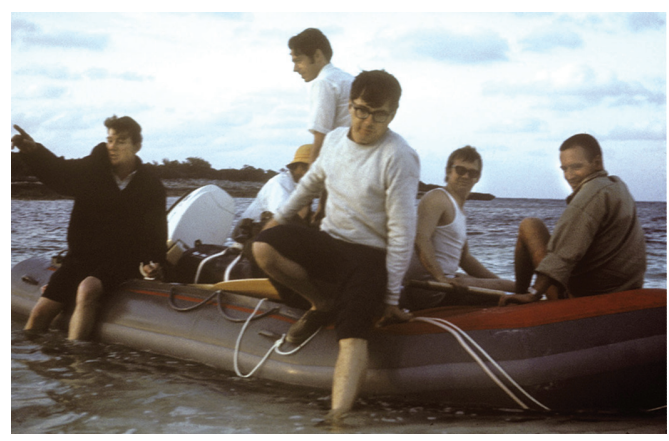

Fig. 2. Acklins Island, Bahamas, 1970. Left to right: Walter Adey, Arthur Dahl, Tom Walker, Klaus Rützler and Arnfried Antonius. Photo by Mary Rice.

(now Association of Marine Laboratories of the Caribbean). The paper was published in the meeting's proceedings in 1976 and was the first report on what Arnfried later named Black Band Disease. From this time on, his work on a wide range of coral ailments became well known among coral reef researchers (Antonius 1976, 1977a, 1977b, 1981a, 1981b, 1984a, 1984b, 1985a, 1985b, 1987, 1988a, 1988b, 1989, 1991, 1993, 1995a, 1995b, 1995c, 1995d, 1996, 1998, 1999a, 1999b, 2000a, 2000b, Dahl et al. 1974, Antonius et al. 1978, 1990, Dodge et al. 1983, Rützler et al. 1983, Antonius \& Riegl 1997, 1998, Antonius \& Ballesteros 1998, Antonius \& Lipscomb 2000, Verlaque et al. 2000, Antonius \& Alfonso-Carrillo 2001, Riegl \& Antonius 2003). His contributions to our understanding of specific coral diseases and afflictions were summarized by Richardson (2011), and the complete bibliography is included here.

Arnfried accepted a new position in Jeddah, Saudi Arabia, and moved there with Jocelyn in 1980. He joined the faculty at the King Abdulaziz University and began expanding his observations on coral pathology to the Red Sea and the Indo-Pacific (Antonius 1984a). In 1989, Arnfried and his family, now with daughter Anya, moved on to Austria where he continued his studies, gave lectures, was actively involved in the establishment of marine parks in Sinai, Egypt (late 1990s), 
regularly visited the Caribbean, and guided graduate students until 2002. He concluded his research endeavors with the examination of "coral-killing" red algae and ciliates. After sudden health complications, which emerged in late December 2009, Arnfried passed away on January $13^{\text {th }}, 2010$.

The coral pathology session of the $35^{\text {th }}$ AMLC scientific meeting in Costa Rica (2011) was dedicated to Arnfried Antonius, as proposed by Jorge Cortés. Arnfried's contribution in kicking off awareness about coral pathology, amidst the widespread initial indifference about the topic among his peers, is well known and so my account does not pivot on his first descriptions of Black Band Disease. Instead, I intended to outline the path he walked throughout his years of involvement in marine science, many of which he spent in the Caribbean (Fig. $3)$. Fourteen years ago, at the $28^{\text {th }}$ AMLC meeting, which was also held at the CIMAR in San José, Costa Rica, Arnfried and I spent

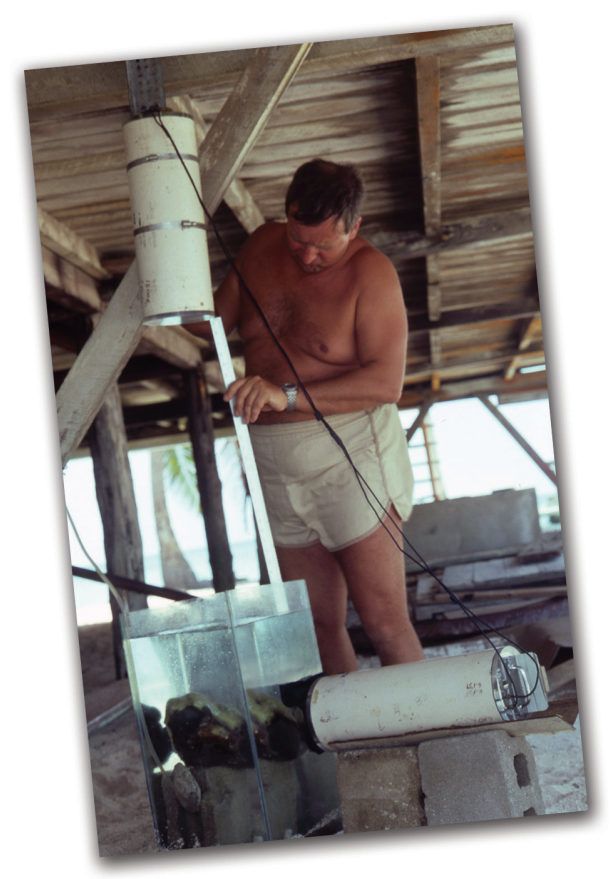

Fig. 3. Arnfried Antonius in 1980, photographing Black Band Disease progressions at Carry Bow Cay. Photo by Klaus Rützler. several hours at a nearby tavern talking about our work experiences, our opinions on the status of coral reefs and research in the Caribbean, among other things. This was not our first or last encounter, but for me it was particularly memorable because it was then that I realized what I appreciated about Arnfried and where I felt a kinship to him. It was his passion for his work and fieldwork in particular, regardless of the logistic and environmental constraints, the popularity of the topic, or the institutional flag, if any, under which work was carried out. $\mathrm{He}$ was a kind man, a good listener, and always ready to help. Arnfried was keenly perceptive of regional differences in environmental ethics and was very objective as to the importance of marine research. It is thus not surprising that he had a great sense of humor and never took himself too seriously, or the science circus with which we often surround ourselves.

\section{ACKNOWLEDGMENTS}

I am deeply grateful to Jocelyn Antonius and Klaus Rützler for the information they have shared with me. Klaus Rützler provided the photos, and Bernhard Riegl shared a few accounts form Sinai. My thanks also go to Jorge Cortés for asking me to write this article, which I happily accepted.

\section{REFERENCES}

Antonius, A. 1965. Methodischer Beitrag zur mikroskopischen Anatomie und graphischen Rekonstruktion sehr kleiner zoologischer Objekte. Mikroskopie 20: 145-153.

Antonius, A. 1967. Neue Convolutidae (Turbellaria acoela) aus dem Roten Meer. Ph.D.Thesis, University of Vienna, Vienna, Austria.

Antonius, A. 1968. The distribution of stony corals in the Gulf of Cariaco, Venezuela, an area of extreme environmental conditions. Abstract, Assoc. Island Marine Labs Carib. 8: 3.

Antonius, A. 1971. Das Acanthaster Problem im Pazifik. Int. Rev. Ges. Hydrobiol. 56: 283-313. 
Antonius, A. 1972. Occurrence and distribution of stony corals (Anthozoa and Hydrozoa) in the vecinity of Santa Marta, Colombia. Mitt. Inst. Colombo-Alemán Invest. Cient. Punta Betín, 6: 89-103.

Antonius, A. 1976. New observations on coral destruction in reefs. Assoc. Island Marine Labs Carib.10: 17.

Antonius, 1977a. Kranke Korallen: Riffzerstörung. Umschau Wiss. Techn.76: 493-494.

Antonius, 1977b. Coral mortality in reefs: a problem for science and management. Proc. $3^{\text {rd }}$ Inst. Coral Reef Symp., Miami 2: 617-623.

Antonius, A. 1980. Occurrence and distribution of stony corals in the Gulf of Cariaco, Venezuela. Int. Rev. Ges. Hydrobiol., 65: 321-338.

Antonius, A. 1981a. Coral reef pathology: a review. Proc. $4^{\text {th }}$ Int. Coral Reef Symp., Manila 2: 3-6.

Antonius, A. 1981b. The "band" diseases in coral reefs. $4^{\text {th }}$ Int. Coral Reef Symp., Manila 3: 7-14.

Antonius, A. 1984a. Korallenkrankheiten: erstmals im Indo-Pazifik nachgewiesen. Umschau Wiss. Techn. 84: 706-798.

Antonius, A. 1984b. Human impacts on corals in fringing reefs near Jeddah. Symp. Coral Reef Environment, Fac. Mar. Sci., King Abdulaziz Univ., Jeddah, Saudi Arabia, 1984: 363-389.

Antonius, A. 1985a. Coral diseases in the Indo-Pacific: a first record. P.S.Z.N.I: Mar. Ecol. 6: 197-218.

Antonius, A. 1985b. Black band disease infection experiments on hexacorals and octocorals. Proc $5^{\text {th }}$ Int Coral Reef Symp., Tahiti 6: 155-161.

Antonius, A. 1987. Survey of Red Sea coral health 1-Jeddah to Jizan. Proc. $10^{\text {th }}$ Symp. Biological Aspects of Saudia Arabia, Saudi Biol. Soc. 1987: 363-389.

Antonius, A. 1988a. Distribution and dynamics of coral diseases in the eastern Red Sea. Proc. $6^{\text {th }}$ Int. Coral Reef Symp., Townsville 2: 293-297.

Antonius, A. 1988b. Black Band Disease behavior on Red Sea reef corals. Proc. $6^{\text {th }}$ Int. Coral Reef Symp., Townsville 3: 145-150.

Antonius, A. 1989. Coral pathology and sewater pollution in the eastern Red Sea. Int. Soc. Reef Stud., Annual Meeting, Abstracts: 25 .
Antonius, A. 1991. La maladie de coreaux dans les zonestests no. 1 et 2. In J. Muller (ed.). Etude des ecosystemes littoreaux de Maurice. ECC projet 946/89, 5 : 145-165.

Antonius, A. 1993. Coral reef health in Mauritius. Proc. $1^{\text {st }}$ European Meet. Int. Soc. Reef Stud., Vienna. Austria. Abstracts: 2 .

Antonius, A. 1995a. Pathologic syndromes as indicators on reef corals: a review. Proc. $2^{\text {nd }}$ European Meet. Int. Soc. Reef Stud., Publ. Serv. Geol. Lux. 29: 161-169.

Antonius, A. 1995b. Pathologic syndromes as indicators on reef corals: field methods. Proc. $2^{\text {nd }}$ European Meet. Int. Soc. Reef Stud., Publ. Serv. Geol. Lux. 29: 231-235.

Antonius, A. 1995c. Sinai coral reef health survey I: first spot checks. Rep. Ras Mohamed Nat. Park Serv., Sinai, Egypt. 7 p.

Antonius, A. 1995d. Incidence and prevalence of coral diseases on coral reefs: what progress in research? Coral Reefs 14: 224.

Antonius, A. 1996. Sinai coral reef health survey II: Gulf of Aqaba, Straits of Tiran and Ras Mohamed. Rep. Ras Mohamed Nat. Park Serv., Sinai, Egypt. 19 p.

Antonius, A. 1998. Some parameters of Indo-Pacific and Caribbean reef health: changes over time. Abtsarct, Proc. $3^{\text {rd }}$ European Meet. Int. Soc. Reef Stud., Perpignan, France.

Antonius, A. 1999a. Metapeyssonnelia corallepida, a new coral-killer red alga on Caribbean reefs. Coral Reefs 18: 301 .

Antonius, A. 1999b. Halofolliculina corallasia, a new coral-killing ciliate on Indo-Pacific reefs. Coral Reefs 18: 300 .

Antonius, A. 2000a. Biogeography of old and new coral diseases. Abstract, $9^{\text {th }}$ Int Coral Reef Symp. Bali: 280.

Antonius, A. 2000b. Bioerrosion on Indo-Pacific reef corals: the skeleton eroding band (SEB) disease. Abstract, $3^{\text {rd }}$ Int. Bioerosion Workshop, Barcelona: 13.

Antonius, A. \& J. Alfonso-Carrillo. 2001. Pneophyllum conicum killing reef corals in Mauritius: A new IndoPacific syndrome? Bull. Mar. Sci. 69: 613-618.

Antonius, A. \& E. Ballesteros. 1998. Epizoism: a new threat to coral health in Caribbean reefs. Rev. Biol. Trop. 46 (Supl. 5): 145-156. 
Antonius, A. \& D. Lipscomb. 2000. First protozoan coralkiller identified in the Indo-Pacific. Atoll Res. Bull. 481: 1-21.

Antonius, A. \& B. Riegl. 1997. A possible link between coral diseases and a corallivorous snail (Drupella cornus) outbreak in the Red Sea. Atoll Res. Bull. 447: 1-9.

Antonius A. \& B. Riegl. 1998. Coral diseases and Drupella cornus invasion in the northern Red Sea. Coral Reefs $17: 84$.

Antonius, A. \& A. Weiner. 1982. Reefs under fire. P.S.Z.N.I: Mar. Ecol. 3: 255-277.

Antonius, A., A. Weiner, J. Halas \& E. Davidson. 1978 Looe Key reef resource inventory. Florida Reef Foundation Report to US Dept. Commerce, NOAA, 63 p.

Antonius, A., G. Scheer \& C. Bouchon. 1990. Corals of the eastern Red Sea. Atoll Res. Bull. 334: 1-22.

Dahl, A.L., I.G. Macintyre, \& A. Antonius. 1974. A comparative survey of coral reef research sites. Atoll Res. Bull. 172: 37-120.
Dodge, R.E., A. Logan \& A. Antonius. 1983. Quantitative reef assessment studies in Bermuda: a comparison of methods and preliminary results. Bull. Mar. Sci. 32: $745-760$.

Richardson, L.L. 2011. Arnfried Antonius, coral diseases and the AMLC. Rev. Biol. Trop. XX (Suppl.X): X-X.

Riegl, B. \& A. Antonius. 2003. Halofolliculina skeleton eroding band (SEB): a coral disease with fossilization potential? Coral Reefs 22: 48.

Rützler, K. 2009. Caribbean Coral Reef Ecosystems: Thirty-five years of Smithsonian marine science in Belize. Proc. Smithsonian Mar. Sci. Symp., Smithsonian Contr. Mar. Sci. 38: 43-71.

Rützler, K., D.L. Santavy \& A. Antonius. 1983. The black band disease of reef corals 3: Distribution, ecology and course of disease. Mar. Ecol. 4: 329-358.

Verlaque, M., E. Ballesteros \& A. Antonius. 2000. Metapeyssonnelia corallepida sp. nov. (Peyssonneliacea, Rhodophyta), an Atlantic encrusting red alga overgrowing corals. Bot. Mar. 43: 191-200. 



\section{Arnfried Antonius (1934 - 2010): una semblanza}

Sascha C.C. Steiner ${ }^{1,2}$

1. Trostgasse 16, 2500 Baden, Austria

2. Institute for Tropical Marine Ecology Inc., P.O. Box 944, Roseau, Dominica; scc.steiner@itme.org

Received 28-VI-2011. C Corrected 20-IX-2011. Accepted 20-XII-2011.

Arnfried Antonius (Fig. 1) estudio Zoología, Paleontología y Etnología en la Universidad de Viena, en Austria, pero luego centró su atención en Ecología y Reconstrucción gráfica micro-anatómica de invertebrados marinos (Antonius 1965). Completó su doctorado en 1967 con una tesis sobre Platyhelminthes retorcidos del Mar Rojo (Antonius 1967). El Instituto de Biología Marina de Rovinj (antes Yugoslavia, ahora Croacia) y el Museo de Historia Natural de Venecia en Italia eran algunos de los lugares en los que llevaba a cabo su trabajo. Más adelante viaja a Italia donde explora la región de Apulia mientras ayuda a Michele Sarà de la Universidad de Bari a buscar un lugar para establecer una estación para estudiar el litoral. Tres lugares reúnen las condiciones, pero antes de elegir uno $\mathrm{M}$. Sarà se trasladó a Génova y se abandonó el proyecto. En 1968, siguiendo su deseo de ver el mundo y explorarlo, Arnfried acepta la invitación del hidrobotánico Fritz Gessner de la Universidad de Oriente, en Venezuela, para trabajar en un nuevo Centro de Investigación en Cumaná, y pasa a ser profesor de esa institución. Aunque contaba con financiamiento - significativo para la época- el Centro no tenía ni laboratorio de histología ni microscopios, así que Arnfried empieza a trabajar con animales "más grandes", concretamente con corales duros, tanto del Golfo de Cariaco, sitio que posee una zona de afloramiento muy interesante

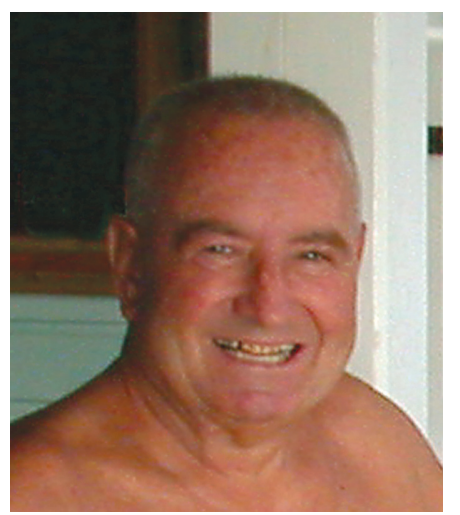

Fig. 1. Arnfried Antonius en Cayo Carry Bow en 2001. Detalle de una foto por Mike Carpenter.

(Antonius 1968, 1980), como de Colombia (Antonius 1972). En 1968, en el IV Congreso Latinoamericano de Zoología celebrado en Caracas, conoce a Jocelyn López Montes de Oca, que en ese entonces estudiaba quitones en la Universidad Autónoma de Santo Domingo (siendo la primera persona que se gradua en biología marina en esa Universidad) y posteriormente se casan. En 1969, la aparición de cantidades masivas de Acanthaster planci en el Pacífico genera pánico pero también fondos para evaluar la situación. Arnfried recibe una invitación para viajar a Micronesia y participar de este esfuerzo en las islas de Ifaluk y Woleei (Antonius 1971). 
En 1972, Arnfried visita a Klaus Rützler -colega suyo también graduado de la Universidad de Viena-, en el Museo de Historia Natural del Instituto Smithsoniano en Washington D.C., para examinar las colecciones de corales. Anteriormente ya habían colaborado en estudios sobre arrecifes coralinos y la visita se traduce en una posición post-doctoral (Fig. 2). También marca el inicio de la continuada participación de Arnfried en las ciencias marinas del Caribe que comienza con estudios sobre los arrecifes de Belice (British Honduras en ese momento) y con un acontecimiento histórico, involucrando la momentánea pérdida del rumbo por parte de la tripulación. Arnfried y Klaus debían fletar un bote para recuperar un bote de hule, un motor, un compresor y otros aparatos que habían sido dejados en el Arrecife de Glover por un grupo de biólogos y geólogos, jóvenes y entusiastas, que estaban convencidos de que iban a recibir fondos para establecer una estación de investigación en uno de los pocos atolónes del Caribe. Su convencimiento derivaba de los amplios estudios que habían llevado a cabo en la región y del hecho de que se habían reunido con representantes de unas 40 instituciones en un taller cuyo objetivo era formalizar la propuesta para dicha estación (Rützler 2009). El Arrecife de Glover es un atolón situado a unos $20 \mathrm{~km}$ al este de la barrera arrecifal de Belice. La propuesta del grupo de investigadores era estudiar el Arrecife de Glover por un período de 10 años, para después compararlo a un un atolón del Pacífico, pero el financiamiento no llegó nunca. Tras retirar el equipo, Arnfried y Klaus emprendieron el regreso. El capitán perdió momentáneamente el rumbo y no encontró la entrada por Cayo Tabaco. Encontró, sin embargo, otro pasaje, al lado del cual había una islita. Arnfried, Klaus y el resto de la tripulación desembarcaron en el lugar. La isla estaba desierta, pero los recibió un rótulo que decía "Bienvenidos a Cayo Carrie Bow". El resto es historia, como se dice en inglés, y está bien documentado en el resumen de los estudios que se han llevado a cabo en la Estación Biológica de Cayo Carrie Bow, desde su establecimiento en 1972 (Rützler 2009).

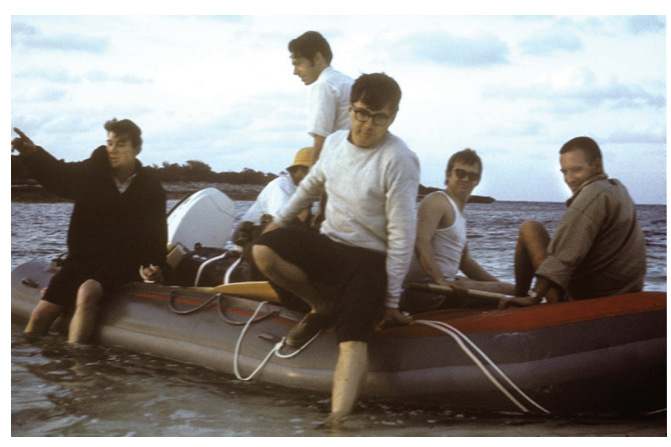

Fig. 2. Isla Acklins, Bahamas, 1970. De izquierda a derecha: Walter Adey, Arthur Dahl, Tom Walker, Klaus Rützler and Arnfried Antonius. Foto por Mary Rice.

En 1973 Arnfried empieza a trabajar para la Harbor Branch Foundation en Fort Pierce, Florida. Ya había comenzado a ponerle atención a la degradación de los arrecifes en todas sus formas y apariencias, suplementando las observaciones de campo con experimentos y métodos de patología. Ese mismo año cofunda la Florida Reef Foundation y presenta su primer artículo sobre algas que matan corales durante la X Reunión Científica de la Asociación de Laboratorios Marinos Isleños del Caribe (ahora Asociación de Laboratorios Marinos del Caribe). El trabajo se publica en 1976 en las memorias de la reunión. Los trabajos que publica de ahí en adelante constituyen lectura obligatoria entre los investigadores de arrecifes coralinos (Antonius 1976, 1977a, 1977b, 1981a, 1981b, 1984a, 1984b, 1985a, 1985b, 1987, 1988a, 1988b, 1989, 1991, 1993, 1995a, 1995b, 1995c, 1995d, 1996, 1998, 1999a, 1999b, 2000a, 2000b, Dahl et al. 1974, Antonius et al. 1978, 1990, Dodge et al. 1983, Rützler et al. 1983, Antonius \& Riegl 1997, 1998, Antonius \& Ballesteros 1998, Antonius \& Lipscomb 2000, Verlaque et al. 2000, Antonius \& Alfonso-Carrillo 2001, Riegl \& Antonius 2003). Sus contribución a nuestro conocimiento de enfermedades especificas de los corales fueron resumidos por Richardson (2011) y aquí se incluye la bibliografía completa.

En 1980, Arnfried y Jocelyn se mudan a Jeddah, Arabia Saudita. Arnfried se une 
al cuerpo docente de la Universidad King Abdulaziz y amplía el ámbito de sus estudios sobre patologías coralinas al Mar Rojo y al Indo-Pacífico (Antonius 1984a). En 1989, él y su familia, ahora con una hija, Anya, regresan a Austria, donde el investigador prosigue sus estudios, imparte clases, colabora en el establecimiento de parques marinos en el Sinaí, en Egipto (finales de la década de 1990), continúa visitando el Caribe regularmente y ofrece consejos a estudiantes de posgrado hasta el 2002. Concluye sus esfuerzos de investigación estudiando algas rojas y organismos ciliados que colonizan y matan corales. Su salud se complica súbitamente a finales de diciembre de 2009 y muere el 13 de enero de 2010.

La sesión sobre patología de corales de la XXXV Reunión Científica de la ALMC celebrada en Costa Rica en el 2011 estuvo dedicada a Arnfried Antonius, a petición de Jorge Cortés. La contribución de Arnfried al

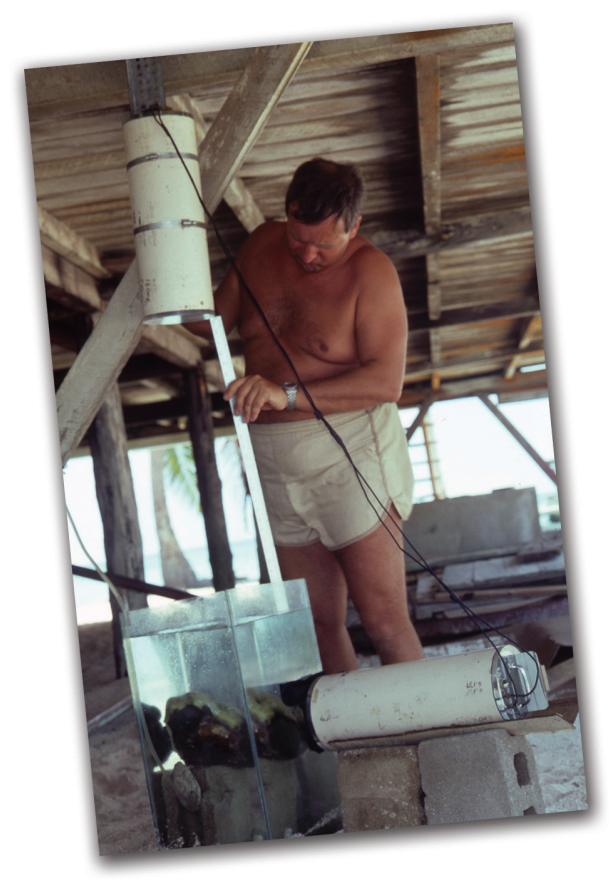

Fig. 3. Arnfried Antonius en 1980, tomando fotos de la progresión de la enfermedad de banda negra en Cayo Carry Bow. Foto por Klaus Rützler. estudio de las patologías coralinas, pese a la indiferencia con que el tema era mirado en un inicio por sus colegas, es de sobra conocida. De ahí que mis comentarios no hayan girado alrededor de sus primeras descripciones de la "enfermedad de banda negra" que afecta a los corales. Mi intención ha sido, más bien, recorrer la ruta tomada por Arnfried durante los años que dedicó a las ciencias marinas, muchos de los cuales los pasó en el Caribe (Fig. 3). Hace catorce años, durante la XXVIII Reunión Científica de la ALMC, que también se efectuó en el CIMAR en San José de Costa Rica, Arnfried y yo pasamos varias horas en una taberna hablando de nuestras experiencias de trabajo, intercambiando opiniones sobre el estado de los arrecifes y comentando sobre la investigación marina en el Caribe, entre otras cosas. No fue ni nuestro primer encuentro ni el último, pero sí un encuentro memorable, porque ahí me di cuenta de qué era lo que yo más apreciaba de Arnfried y por qué sentía que "éramos de los mismos": era su pasión por el trabajo, sobre todo el trabajo de campo, al que se entregaba sin importar la popularidad del tema, los retos logísticos o ambientales o la bandera institucional, si es que la había. Era un hombre amable, sabía escuchar y siempre estaba dispuesto a ayudar. Su agudeza mental le permitió percibir claramente las diferencias regionales en materia de ética ambiental y ver con objetividad la importancia de las investigaciones marinas. Por eso, no sorprende que tuviera tan buen sentido del humor y que nunca se tomara demasiado en serio, ni a sí mismo, ni al circo científico del que a menudo solemos rodearnos.

\section{AGRADECIMIENTOS}

Quedo profundamente agradecido con Jocelyn Antonius y con Klaus Rützler por la información que compartieron conmigo. Klaus Rützler aportó las fotos y Bernhard Riegl compartió conmigo muchas de las experiencias vividas en el Sinaí. Gracias también a Jorge Cortés, que me pidió que escribiera este artículo, lo que acepté con gran alegría. 


\section{BIBLIOGRAFIA}

Antonius, A. 1965. Methodischer Beitrag zur mikroskopischen Anatomie und graphischen Rekonstruktion sehr kleiner zoologischer Objekte. Mikroskopie 20: 145-153.

Antonius, A. 1967. Neue Convolutidae (Turbellaria acoela) aus dem Roten Meer. Ph.D. Thesis, University of Vienna, Vienna, Austria.

Antonius, A. 1968. The distribution of stony corals in the Gulf of Cariaco, Venezuela, an area of extreme environmental conditions. Abstract, Assoc. Island Marine Labs Carib. 8: 3.

Antonius, A. 1971. Das Acanthaster Problem im Pazifik. Int. Rev. Ges. Hydrobiol. 56: 283-313.

Antonius, A. 1972. Occurrence and distribution of stony corals (Anthozoa and Hydrozoa) in the vecinity of Santa Marta, Colombia. Mitt. Inst. Colombo-Alemán Invest. Cient. Punta Betín, 6: 89-103.

Antonius, A. 1976. New observations on coral destruction in reefs. Assoc. Island Marine Labs Carib.10: 17.

Antonius, 1977a. Kranke Korallen: Riffzerstörung. Umschau Wiss. Techn.76: 493-494.

Antonius, 1977b. Coral mortality in reefs: a problem for science and management. Proc. $3^{\text {rd }}$ Inst. Coral Reef Symp., Miami 2: 617-623.

Antonius, A. 1980. Occurrence and distribution of stony corals in the Gulf of Cariaco, Venezuela. Int. Rev. Ges. Hydrobiol., 65: 321-338.

Antonius, A. 1981a. Coral reef pathology: a review. Proc. $4^{\text {th }}$ Int. Coral Reef Symp., Manila 2: 3-6.

Antonius, A. 1981b. The "band" diseases in coral reefs. $4^{\text {th }}$ Int. Coral Reef Symp., Manila 3: 7-14.

Antonius, A. 1984a. Korallenkrankheiten: erstmals im Indo-Pazifik nachgewiesen. Umschau Wiss. Techn. 84: 706-798.

Antonius, A. 1984b. Human impacts on corals in fringing reefs near Jeddah. Symp. Coral Reef Environment, Fac. Mar. Sci., King Abdulaziz Univ., Jeddah, Saudi Arabia, 1984: 363-389.

Antonius, A. 1985a. Coral diseases in the Indo-Pacific: a first record. P.S.Z.N.I: Mar. Ecol. 6: 197-218.

Antonius, A. 1985b. Black band disease infection experiments on hexacorals and octocorals. Proc $5^{\text {th }}$ Int Coral Reef Symp., Tahiti 6: 155-161.
Antonius, A. 1987. Survey of Red Sea coral health. 1-Jeddah to Jizan. Proc. $10^{\text {th }}$ Symp. Biological Aspects of Saudia Arabia, Saudi Biol. Soc. 1987: 363-389.

Antonius, A. 1988a. Distribution and dynamics of coral diseases in the eastern Red Sea. Proc. $6^{\text {th }}$ Int. Coral Reef Symp., Townsville 2: 293-297.

Antonius, A. 1988b. Black Band Disease behavior on Red Sea reef corals. Proc. $6^{\text {th }}$ Int. Coral Reef Symp., Townsville 3: 145-150.

Antonius, A. 1989. Coral pathology and sewater pollution in the eastern Red Sea. Int. Soc. Reef Stud., Annual Meeting, Abstracts: 25.

Antonius, A. 1991. La maladie de coreaux dans les zonestests no. 1 et 2. In J. Muller (ed.). Etude des ecosystemes littoreaux de Maurice. ECC projet 946/89, 5 : 145-165.

Antonius, A. 1993. Coral reef health in Mauritius. Proc. $1^{\text {st }}$ European Meet. Int. Soc. Reef Stud., Vienna. Austria. Abstracts: 2.

Antonius, A. 1995a. Pathologic syndromes as indicators on reef corals: a review. Proc. $2^{\text {nd }}$ European Meet. Int. Soc. Reef Stud., Publ. Serv. Geol. Lux. 29: 161-169.

Antonius, A. 1995b. Pathologic syndromes as indicators on reef corals: field methods. Proc. $2^{\text {nd }}$ European Meet. Int. Soc. Reef Stud., Publ. Serv. Geol. Lux. 29: 231-235.

Antonius, A. 1995c. Sinai coral reef health survey I: first spot checks. Rep. Ras Mohamed Nat. Park Serv., Sinai, Egypt. 7 p.

Antonius, A. 1995d. Incidence and prevalence of coral diseases on coral reefs: what progress in research? Coral Reefs 14: 224.

Antonius, A. 1996. Sinai coral reef health survey II: Gulf of Aqaba, Straits of Tiran and Ras Mohamed. Rep. Ras Mohamed Nat. Park Serv., Sinai, Egypt. 19 p.

Antonius, A. 1998. Some parameters of Indo-Pacific and Caribbean reef health: changes over time. Abtsarct, Proc. $3^{\text {rd }}$ European Meet. Int. Soc. Reef Stud., Perpignan, France.

Antonius, A. 1999a. Metapeyssonnelia corallepida, a new coral-killer red alga on Caribbean reefs. Coral Reefs 18: 301 .

Antonius, A. 1999b. Halofolliculina corallasia, a new coral-killing ciliate on Indo-Pacific reefs. Coral Reefs 18: 300 . 
Antonius, A. 2000a. Biogeography of old and new coral diseases. Abstract, $9^{\text {th }}$ Int Coral Reef Symp. Bali: 280.

Antonius, A. 2000b. Bioerrosion on Indo-Pacific reef corals: the skeleton eroding band (SEB) disease. Abstract, $3^{\text {rd }}$ Int. Bioerosion Workshop, Barcelona: 13.

Antonius, A. \& J. Alfonso-Carrillo. 2001. Pneophyllum conicum killing reef corals in Mauritius: A new IndoPacific syndrome? Bull. Mar. Sci. 69: 613-618.

Antonius, A. \& E. Ballesteros. 1998. Epizoism: a new threat to coral health in Caribbean reefs. Rev. Biol. Trop. 46 (Supl. 5): 145-156.

Antonius, A. \& D. Lipscomb. 2000. First protozoan coralkiller identified in the Indo-Pacific. Atoll Res. Bull. 481: 1-21.

Antonius, A. \& B. Riegl. 1997. A possible link between coral diseases and a corallivorous snail (Drupella cornus) outbreak in the Red Sea. Atoll Res. Bull. 447: 1-9.

Antonius A. \& B. Riegl. 1998. Coral diseases and Drupella cornus invasion in the northern Red Sea. Coral Reefs 17:84.

Antonius, A. \& A. Weiner. 1982. Reefs under fire. P.S.Z.N.I: Mar. Ecol. 3: 255-277.

Antonius, A., A. Weiner, J. Halas J. \& E. Davidson. 1978. Looe Key reef resource inventory. Florida Reef Foundation Report to US Dept. Commerce, NOAA, 63 p.
Antonius, A., G. Scheer \& C. Bouchon. 1990. Corals of the eastern Red Sea. Atoll Res. Bull. 334: 1-22.

Dahl, A.L., I.G. Macintyre, \& A. Antonius. 1974. A comparative survey of coral reef research sites. Atoll Res. Bull. 172: 37-120.

Dodge, R.E., A. Logan \& A. Antonius. 1983. Quantitative reef assessment studies in Bermuda: a comparison of methods and preliminary results. Bull. Mar. Sci. 32: 745-760.

Richardson, L. L. 2011. Arnfried Antonius, coral diseases and the AMLC. Rev. Biol. Trop. XX (Suppl.X): X-X.

Riegl, B. \& A. Antonius. 2003. Halofolliculina skeleton eroding band (SEB): a coral disease with fossilization potential? Coral Reefs 22: 48.

Rützler, K. 2009. Caribbean Coral Reef Ecosystems: Thirty-five years of Smithsonian marine science in Belize. Proc. Smithsonian Mar. Sci. Symp., Smithsonian Contr. Mar. Sci. 38: 43-71.

Rützler, K., D.L. Santavy \& A. Antonius. 1983. The black band disease of reef corals 3: Distribution, ecology and course of disease. Mar. Ecol. 4: 329-358.

Verlaque, M., E. Ballesteros \& A. Antonius. 2000. Metapeyssonnelia corallepida sp. nov. (Peyssonneliacea, Rhodophyta), an Atlantic encrusting red alga overgrowing corals. Bot. Mar. 43: 191-200. 
